\title{
Circulação secundária atípica em meandro fluviomarinho como parâmetro hidrodinâmico em ecossistema aquático amazônico
}

A circulação lateral $(\mathrm{CL})$ em curvaturas de rios é influenciada pela morfologia dos canais, sendo relevante em processos de mistura que interferem na variação da qualidade da água. O objetivo desta pesquisa é avaliar $\mathrm{CL}$ a partir do escoamento hidrodinâmico, curvatura e geometria de um rio fluviomarinho amazônico. $\mathrm{O}$ escoamento foi quantificado em uma seção transversal do Rio Araguari-Amapá/Brasil cujas características geométricas são consideradas ideais para testar CL em trechos curvos (raio de curvatura $\sim 5900 \mathrm{~m}$ ). As medidas ocorreram trimestralmente durante ciclos de marés semidiunos (12,5h), em Junho, Setembro e Dezembro/2013 e Março/2014 e 2015, utilizando um perfilador de corrente acústico (ADCP). Este trecho apresenta perturbação hidrodinâmica de um afluente próximo a montante de uma expansão lateral do canal, que varia de 850 para $1750 \mathrm{~m}$ e, posteriormente, de 1750 para $2900 \mathrm{~m}$. Os perfis hidrodinâmico e geométrico foram processados nos softwares Matlab e Tecplot, permitindo avaliar e comparar o padrão das linhas de corrente do escoamento formador da $\mathrm{CL}$ com informações similares da literatura. Os resultados indicaram ausência de um padrão helicoidal típico. Assim, na maré vazante as linhas de corrente fluem da margem externa para a interna em descendente da superfície para o fundo. Na maré enchente, ocorreu o oposto, com linhas de corrente fluindo da margem interna para a externa em ascendência. Testes estatísticos de hipótese mostraram que fatores físicos além da curvatura (rio a montante, expansão geométrica, fases das marés e sazonalidade) restringiram a formação do padrão helicoidal esperado na seção de estudo. Concluímos que apesar destes fatores a intensidade do fluxo secundário foi mais influenciada pela fase da maré, onde o menor valor de $\mathrm{Vy}\left(\left(\right.\right.$ Transversal)/Vx(Longitudinal) ocorreu na fase vazante ( $\left.{ }^{\sim} 0,091\right)$ e o maior na fase enchente $(\sim 0,169)$. Assim, $C L$ variou no intervalo $[\sim 10 \%=C L=\sim 17 \%]$, representando uma considerável forçante física de mistura no escoamento, mesmo em rios com curvaturas relativamente baixas como a apresentada no Rio Araguari no presente estudo.

Palavras-chave: Curvaturas de canais; Fluxo secundário; Hidrodinâmica fluvial; Morfologia fluvial; Fluxo helicoidal.

\section{Atypical secondary circulation in fluvial-marine meander as a hydrodynamic parameter in an Amazonian aquatic ecosystem}

\begin{abstract}
Lateral circulation (LC) in river curves is influenced by the morphology of the channels, being relevant in mixing processes that interfere in the variation of wate quality. The objective of this research is to evaluate LC from the hydrodynamic flow, curvature and geometry of an Amazonian fluviomarine river. The flow was quantified in a cross-section of the Araguari-Amapá River/Brazil whose geometric characteristics are considered ideal for testing LC in curved stretches (curvature radius $~ 5900 \mathrm{~m}$ ). The measurements occurred quarterly during semidiune tide cycles (12.5h) in June, September and December/2013, March/2014 and 2015, using an acoustic doppler current profiler (ADCP). This stretch presents hydrodynamic disturbance of a tributary close to a lateral expansion of the canal, which ranges from 850 to $1750 \mathrm{~m}$ and, later, from 1750 to $2900 \mathrm{~m}$. The hydrodynamic and geometric profiles were processed in the Matlab and Tecplot software, allowing to evaluate and compare the pattern of the current lines of the LC-form flow with similar information in the literature. The results indicated absence of a typical helical pattern. Thus, at the ebb tide the current lines flow from the outer to the inner margin descending from the surface to the bottom. At the flood tide, the opposite occurred, with current lines flowing from the inner to the outer margin in ascendancy. Statistical hypothesis tests showed that physical factors beyond curvature (upstream river, later geometric expansion, tidal phases and seasonality) restricted the formation of the helical pattern expected in the study section. We conclude that, despite these factors, the intensity of the secondary flow was more influenced by the tidal phase, where the lowest $\mathrm{Vy}(($ Transverse)/Vx(Longitudinal) value occurred in the ebb phase ( $\sim 0.091)$ and the highest in the flood phase ( $\sim 0.169)$. Therefore, LC varied in the range [ $\sim 10 \%=$ $\mathrm{CL}=\sim 17 \%]$, representing a considerable physical prompter of mixing in the flow, even in rivers with relatively low curvatures such as that presented in the Araguari River in the present study.
\end{abstract}

Keywords: River Bends; Secondary Flow; River Hydrodynamics; River Morphology; Helical Flow.

Topic: Engenharia de Recursos Hídricos

Reviewed anonymously in the process of blind peer.
Received: 05/03/2021

Approved: 23/03/2021
Eldo Silva dos Santos (iD)

Universidade Federal do Amapá, Brasil

http://lattes.cnpq.br/2437240899117074

http://orcid.org/0000-0001-6886-6298

santos eldo@yahoo.com.br

Alan Cavalcanti da Cunha (iD

Universidade Federal do Amapá, Brasil

http://lattes.cnpq.br/2181817533284030

http://orcid.org/0000-0002-1846-9486

alancunha12@gmail.com
Referencing this:

SANTOS, E. S.; CUNHA, A. C.. Circulação secundária atípica em meandro fluviomarinho como parâmetro hidrodinâmico em ecossistema aquático amazônico. Revista Ibero Americana de Ciências Ambientais, v.12, n.3, p.238-254, 2021. DOI: http://doi.org/10.6008/CBPC2179-6858.2021.003.0021

DOI: 10.6008/CBPC2179-6858.2021.003.0021 


\section{INTRODUÇÃO}

A circulação lateral $(\mathrm{CL})$ ocorre perpendicular ao fluxo normal, resultante do balanço entre forças hidrodinâmicas e o atrito com as margens e fundo dos rios. A CL tem papel importante na dinâmica de escoamento e na dispersão de agentes passivos ao escoamento. Destacam-se os hidrossedimentos, nutrientes e até sementes, cada um dos quais sendo extremamente relevantes para manutenção ou alteração da própria geomorfologia dos canais (FRIEDRICHS et al., 1996; KESHAVARZI et al., 2015, CUNHA et al., 2017).

Os padrões de circulação são também importantes nos processos de mistura e transporte de nutrientes nas dimensões vertical e lateral do canal, especialmente do fundo para a superfície e vice-versa. Contudo, essa forçante física é pouco perceptível e, portanto, pouco estuda na literatura. Por outro lado, é relevante por influenciar a produtividade primária dos ecossistemas aquáticos lóticos (ALLAN et al., 2007), sendo também um dos principais fatores modificadores dos leitos dos rios, os quais potencializam efeitos secundários na magnitude do fluxo longitudinal ao longo do escoamento principal. Todos estes efeitos conjuntos, potencialmente, impactam a dinâmica de dispersão em estuários (CHANT, 2002), ajudando a explicar sua alta produtividade e benefícios de serviços ambientais nestes tipos de ambientes (VÖRÖSMARTY et al., 2010).

No entanto, o funcionamento padrão do fluxo depende do momentum do escoamento (energia) e se concentra na margem externa da curva, provocando a erosão desta, enquanto na margem interna ocorre o oposto (deposição), formando as barras. A redistribuição do momentum e o consequente fluxo secundário são suficientes para explicar a formação dos meandros em rios (FAGHERAZZI et al., 2004). A consequência desse processo é um padrão de movimento helicoidal bastante conhecido e estudado (BLANCKAERT et al., 2004; CHANT, 2002; RILEY et al., 2012; SUKHODOLOV et al., 2015). O fluxo através da curva sofre influência da força centrífuga devido à curvatura do canal, promovendo um gradiente de pressão transversal na seção, que induz ao surgimento da circulação secundária (CL). Isto é, a curvatura do canal induz uma superelevação do nível da água próxima à margem externa, causando uma pressão (escoamento lateral) na direção interna da curva, que leva ao movimento helicoidal.

Outro parâmetro relevante é a curvatura do canal. O entendimento da hidrodinâmica em curvaturas de rios apresenta também uma importância ecológica porque pode ajudar a explicar a heterogeneidade espacial da morfologia do leito do rio e o fluxo secundário (SHIELDS et al., 2005; SUKHODOLOV et al., 2015). Além disso, estes padrões de escoamento podem ser induzidos pela curvatura dos canais, criando áreas específicas de remansos hidráulicos que induzem a formação de zonas hidráulicas com seus respectivos padrões de correntezas muito úteis para a sobrevivência de microrganismos aquáticos (SUKHODOLOV et al., 2015; SANTOS, 2017). Por exemplo, algumas análises teórico-experimentais das relações entre característica do fluxo e as densidades de invertebrados mostram que um intervalo ótimo das condições hidráulicas e das características dos substratos em rios estuarinos é fator chave para definir a resposta ecológica das comunidades de invertebrados em relação ao escoamento. Alguns trabalhos já mostraram que os meandros 
dos rios são partes intrínsecas fundamentais dos ecossistemas fluviais (BLETTLER et al., 2008; DOLÉDEC et al., 2007; SANTOS, 2017). Portanto, a hidrodinâmica tende a se relacionar diretamente com os processos de nutrição na cadeia alimentar aquática, seja pelo deslocamento e abrasão de invertebrados bentos, seja indiretamente pela influência da disponibilidade de alimentos e concentração de oxigênio, temperatura e salinidade (ZĂINESCU et al., 2019). Todos estes efeitos combinados afetam a predação, a dispersão de espécies e a sua reprodução. Além disso, a distribuição da velocidade afeta o padrão espaço-temporal das correntes, sendo uma variável física de significativa influência sobre o comportamento das espécies presentes na coluna d'água ou na superfície dos substratos (SUKHODOLOV et al., 2015). Do ponto de vista ecológico, os organismos mais afetados pela turbulência hidrodinâmica, por exemplo, gastam mais energia para se manterem em posição ou se locomoverem em busca de alimentos (KUPILAS et al., 2017). Estes organismos também são influenciados pela disponibilidade de nutrientes transportados na coluna d'água pelas correntes e pelos gases dissolvidos que afetam o metabolismo. Consequentemente afetam também o crescimento desses organismos (KUPILAS et al., 2017).

A característica hidrodinâmica em um trecho específico do rio pode definir quais espécies habitarão aquela seção específica (ALLAN et al., 2007). Por exemplo, os processos ecológicos conhecidos como dispersão hidrocórica ou hidrocoria (CUNHA et al., 2017), são significativamente afetados pelas curvaturas dos rios estuarinos amazônicos, pois em trechos mais sinuosos tendem a "aprisionar" mais as sementes e favorecer mais a dispersão lateral, reduzindo o alcance dispersivo ou hidrocórico das sementes de longa distância (longitudinal). De modo contrário, nos trechos mais retilíneos o alcance das sementes tende a ser maior e com maior inibição da dispersão lateral. E, apesar deste comportamento ser relativamente pouco conhecido nos rios amazônicos apresenta um papel fundamental na geomorfologia desses canais e no seu potencial ecológico. Em última análise, os processos dispersivos nos rios podem tanto controlar a dispersão de sementes quanto definir o local de propagação e formação de novas plantas, podendo inclusive influenciar a formação de novas geomorfologias dos canais (HOWE et al., 1982; MERRITT et al., 2002; NILSSON et al., 2010; CARTHEY et al., 2016).

Deste modo, estas observações justificam estudos específicos sobre o comportamento dos fluxos laterais $(\mathrm{CL})$ ou correntes secundárias em rios estuarinos, principalmente porque há muitas lacunas de conhecimento sobre quais são os efeitos físicos que determinam suas características hidráulicas, especialmente em ecossistemas fluviomarinhos do estuário amazônico (rios com características muito sinuosas). Estas lacunas impõem a necessidade de aplicações de estudos experimentais integrados com modelos numéricos para gerar novos conhecimentos de importância para a gestão e a conservação da biodiversidade tropical (CUNHA et al., 2017).

Além dos fatores ecológicos relacionados ao escoamento propriamente dito, o transporte de sedimentos também está frequente e diretamente correlacionado com os processos de transporte de cargas de nutrientes, características físico-químicas, distribuição de microrganismos dentro da cadeia alimentar e com os ciclos biogeoquímicos (WARD et al., 2013). Mas, normalmente, estes processos hidrodinâmicos podem ser intensificados ou reduzidos nas curvas ou meandros dos rios. O fluxo em trechos fluviomarinhos 
mostra-se mais complexo do que os ambientes lóticos convencionais (rios escoando em uma única direção e que sofrem apenas variações hidrológicas sazonais ou intra-anuais). Isso porque nos estuários ocorrem simultaneamente as oscilações das marés associadas com mudanças diárias de profundidade, velocidade, direção do escoamento e até mudanças dos gradientes de densidade afetados pela salinidade (KIMMERER, 2002; ALLAN et al., 2007).

O conhecimento sobre o fluxo turbulento em meandros de rios ainda está incompleta, com nível de conhecimento nesse campo limitado, normalmente adquirido através de trabalhos experimentais envolvendo modelos físicos e abordagens teóricas (BLANCKAERT, 2011; BLANCKAERT et al., 2004; KASHYAP et al., 2012; MARION-ZARAMELLA, 2006; QIN et al., 2016). Essas lacunas experimentais necessitam ser preenchidas pois são virtualmente desconhecidas na Amazônia. Contudo, estas informações podem alimentar modelos que representem fidedignamente a variabilidade longitudinal do fluxo além de melhorar a adequação de escalas físicas, principalmente ao se extrapolar para rios de grande porte (ABREU et al., 2020), como o trecho fluviomarinho do baixo Rio Araguari/Amapá/Brasil.

Para compreender os processos envolvidos no transporte de matéria associados aos ciclos biogeoquímicos em bacias de estuário (por exemplo, OD, T, TDS e pH) (WARD et al., 2013; 2015; 2016), é importante primeiramente avaliar as estruturas básicas da turbulência e da hidrodinâmica locais. No presente caso, o principal objetivo é entender a importância relativa da CL durante ciclos completos de marés semidiurnas em relação ao fluxo longitudinal (normalmente mais conhecido e estudado) (MARIONZARAMELLA, 2006; WEBSTER, 2010; WARD et al., 2013, 2015, 2016). Desta forma, as medidas detalhadas de campos de velocidade e da corrente em rios são essenciais para a análise dos efeitos das marés nos processos dispersivos em corpos d'água estuarinos. Por outro lado, será também mostrado e discutido que tais medições são complexas e difíceis de executar em campo por causa da característica transiente dos escoamentos nesses ambientes (KAWANISI, 2004; RAZAZ et al., 2015). Isto é, compreender como a estrutura hidrodinâmica se relaciona com CL e é influenciada por meandro em transformação geomorfológica intensa neste trecho da bacia do Rio Araguari (SANTOS et al., 2017).

A presente contribuição é inédita para a região e testa alguns mecanismos e o funcionamento hidráulico na seção natural de estudo bem como suas potenciais implicações na qualidade da água, representando interações físicas em ambientes estuarinos amazônicos induzidas por força centrífuga, estagnação de fluxo e topografia de fundo, que tornam complexas as predições quantitativas de padrões de escoamento, inclusive para uso de modelos hidrodinâmicos de canais naturais (ABREU et al., 2020).

Como objetivos específicos também foram testadas as variáveis explicativas que contribuem com o padrão hidrodinâmico e quais seriam as potenciais consequências ecológico-ambientais após a o progressivo bloqueio da foz do baixo Rio Araguari ocorrido durante os últimos 10 anos de monitoramento (SANTOS et al., 2018).

Nossa hipótese principal é que há pelo menos três níveis de interferência física que podem influenciar o padrão hidrodinâmico do fluxo secundário (CL) na seção de estudo do baixo Rio Araguari: 1) efeito das marés influenciam o sentido, fase do fluxo, e o período hidrológico sazonal (verão, inverno, quadratura ou 
sizígia); 2) proximidade de obstáculos ou agentes físicos, afetando a geomorfologia da seção de estudo do canal, tais como a curvatura e expansão brusca a jusante da seção de estudo; 3 ) interferência do afluente Igarapé Tabaco, próximo e a montante da seção de estudo. Então, nossa análise pressupõe que estes três entes físicos podem atuar simultaneamente na acentuação ou inibição do padrão helicoidal característico das correntes secundárias e, portanto, controladoras da CL.

\section{MATERIAIS E MÉTODOS}

\section{Área de estudo}

O Rio Araguari está localizado na Amazônia Oriental, no Estado do Amapá/Brasil. Sua extensão geográfica é de cerca de $600 \mathrm{~km}$ e sua área de drenagem na bacia hidrográfica é de aproximadamente 42 mil $\mathrm{km}^{2}$. Seu baixo trecho fluviomarinho é influenciado pela hidrografia, alta pluviosidade $(\approx 3.000 \mathrm{~mm}$ ) (OLIVEIRA et al., 2010) e macromarés semidiurnas (h de 4,0 a 6,0 m). A precipitação média anual na bacia é de $\approx 2.389$ $\mathrm{mm}$, com máximas, em algumas áreas da bacia, $\approx 5000 \mathrm{~mm}$ (a noroeste da área de estudo). O período chuvoso abrange os meses entre fevereiro e agosto. O período seco entre setembro e novembro. A temperatura média anual do ar na região do baixo Araguari é de 27,5 으, com pouca variação ao longo do ano.

Na Figura 1 é mostrado um resumo da resposta hidrológica resultante do padrão de distribuição das chuvas na bacia exatamente no médio Rio Araguari. Isto é, da série histórica da vazão na estação fluviométrica de Porto Platon ( $\approx 270 \mathrm{~km}$ a montante da sua foz). Esta estação foi aqui utilizada como referência padrão de controle das vazões estudadas a jusante na bacia hidrográfica e por apresentar a mais completa e consistente série de dados para estudos na bacia do Rio Araguari, considerada a mais importante do Estado do Amapá.

De acordo com a Figura 1 as máximas vazões ocorrem de abril a junho e as mínimas entre outubro e dezembro. Os experimentos de campo, com o uso do ADCP, ocorreram em $270 \mathrm{~km}$ à jusante da estação de Porto Platon. Os períodos de estudo foram de junho/2013 a março/2015, onde foi observada a maior vazão máxima registrada pela estação de 3.415,8 m³ $/ \mathrm{s}$, em 12/maio/2014. Por outro lado, a mínima vazão observada foi de apenas $155,5 \mathrm{~m}^{3} / \mathrm{s}$, em $23 /$ dezembro/2013, sugerindo alta variabilidade hidrológica sazonal.

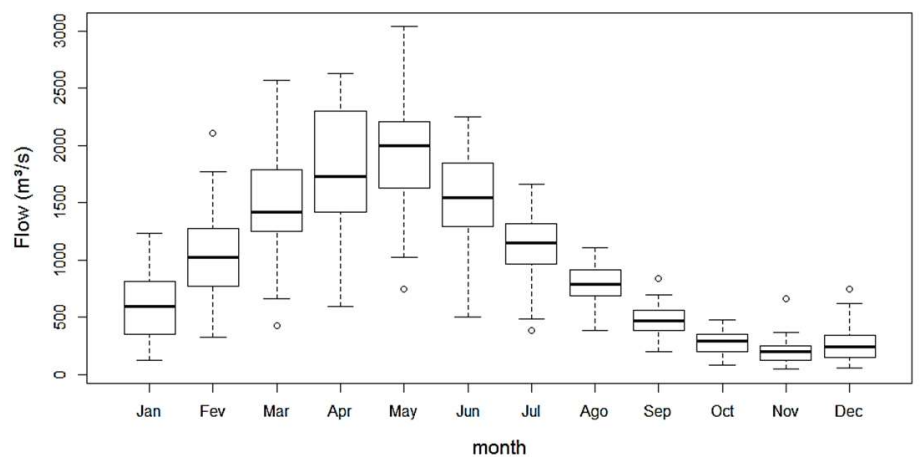

Figura 1: Vazão do Rio Araguari na estação fluviométrica de Porto Platon (042’27”N, 51²6’20”W), de Janeiro/1952 a Agosto/1958 E de Junho/1972 a Novembro/2015. ${ }^{1}$

\footnotetext{
1 A linha central de cada caixa indica a mediana da distribuição, as extremidades inferior e superior da caixa indicam o 1 o e o 30 quartil, respectivamente. As linhas pontilhadas delimitam a distribuição de 1,5 vezes o intervalo interquartil (intervalo entre o 10 e o 30 quartil). Os valores extremos estão indicados pelos círculos.
} 
Nos últimos 10 anos, o Baixo Rio Araguari tem sofrido alterações significativas na sua dinâmica hidrológica por conta da recente conexão com o Rio Amazonas através do Canal Urucurituba, $\approx 80 \mathrm{~km}$ distante da foz do Araguari. Este canal desviou progressivamente significativa fração da água do Araguari para o Amazonas. Algumas evidências têm confirmado que provavelmente este desvio tenha sido a principal causa que provocou o assoreamento da foz além do contínuo e progressivo bloqueio até o seu definitivo fechamento (SANTOS et al., 2018; CUNHA et al., 2018). As medições apresentadas aqui ocorreram justamente no período em que esta modificação na hidrografia do Araguari estava cada vez mais acentuada, provocando mudanças significativas do fluxo original.

Por convenção, neste trabalho, a maré enchente será considerada quando o Rio Araguari estiver fluindo no sentido montante para jusante (em direção ao oceano) e a maré vazante, no sentido contrário. Apesar de ser o percurso natural, essa definição é necessária pois atualmente, o rio "enche" (elevação do nível d'água) quando está fluido em direção à foz e "vaza" quando escoa para montante, por causa da significativa interferência hidráulica causada pela abertura relativamente recente do Canal Urucurituba (SANTOS et al., 2018) (Figura 2).

A seção-chave estudada está distante aproximadamente $60 \mathrm{~km}$ da foz original do rio Araguari no Oceano Atlântico (Figura 2). Este trecho de rio onde se encontra a seção-chave possui raio de curvatura (R) de $5.900 \mathrm{~m}$ e largura de $850 \mathrm{~m}$. Logo após a seção-chave de estudo o canal se expande bruscamente, em até 1.750 de largura a cerca de $2.900 \mathrm{~m}$ a jusante da seção-chave de monitoramento. Neste trecho do rio há significativo efeito de marés semidiurnas. Durante as medições de junho/2014 a março/2015 as variações de elevação do nível da água foram de 0,56 m (março/2014) a 1,42 m (dezembro/2014). A Tabela 1 apresenta um resumo das características físicas da seção-chave nos 6 períodos de medição.


Figura 2: Área de Estudo: A) Trecho do Baixo Araguari no Estado do Amapá; B) Detalhe com a seção-chave de estudo, com os eixos de orientação da velocidade na direção do fluxo ( $\mathrm{Vx}-\mathrm{m} / \mathrm{s}$, positiva no sentido do fluxo vazante) $\mathrm{e}$ perpendicular ao fluxo ( $\mathrm{V} y-\mathrm{m} / \mathrm{s}$, positivo no sentido da margem norte). ${ }^{2}$

\footnotetext{
${ }^{2}$ Estão representadas também o fluxo do rio Araguari ( $Q a-m^{3} / s$, positivo no sentido indicado pela seta) e do Igarapé Tabaco ( $Q t$ - $m^{3} / s$, positivo no sentido indicado pela seta). $D_{1}$ é igual a $850 \mathrm{~m}$ e equivale à largura da seção de medição e $\mathrm{D}_{2}$ (=1750 m) é a largura após a expansão canal do Rio Araguari. A distância entre $D_{1}$ e $D_{2}$ é de $2.900 \mathrm{~m}$.
} 
Tabela 1: Largura medida $(\mathrm{L})$ e profundidade $(\mathrm{H})$ médias na seção de medição ao longo de um ciclo de maré semidiurna. Amplitude da elevação no nível da água (Amp) e do tempo $(\Delta t)$ entre baixa-mar e preamar.

\begin{tabular}{l|l|l|l|l}
\hline Período & $\mathbf{L}(\mathbf{m})$ & $\mathbf{H}(\mathbf{m})$ & $\mathbf{A}(\mathbf{m})$ & $\mathbf{\Delta t}(\mathbf{h})$ \\
\hline Jun-13 & 795,3 & 2.83 & 0,71 & 3,66 \\
\hline Dec-13 & 771,3 & 2.03 & 1,07 & 4,56 \\
\hline Mar-14 & 797,8 & 2.43 & 0,56 & 3,95 \\
\hline Jun-14 & 811,3 & 2.47 & 0,76 & 4,38 \\
\hline Dec-14 & 685,2 & 1.80 & 1,42 & 5,08 \\
\hline Mar-15 & 2,55 & 1,03 & 3,58 \\
\hline
\end{tabular}

\section{Método de extração dos valores experimentais de velocidade}

Os dados hidrodinâmicos obtidos em campo dos componentes da velocidade foram registrados em seis datas entre Junho/2013 e Março/2015 (Tabela 2). Foi utilizado o ADCP (Acoustic Doppler Current Profiller), modelo RiverSurveyor M9, da SonTek/YSI para medições no transecto do Rio Araguari próximo à confluência com o Igarapé Tabaco. O modelo Riversurveyor M9 é um ADCP de múltiplas frequências com um feixe vertical de baixa frequência e algoritmos de configuração que se adaptam (MUELLER et al., 2009). 0 equipamento possui nove feixes sendo quatro com frequência de $1 \mathrm{MHz}$, outros quatro com $3 \mathrm{MHz}$ e um feixe vertical de $500 \mathrm{kHz}$ para medição de profundidade. Através desses feixes, o ADCP mede as três componentes da velocidade ( $V e, V n, V z)$ em células individuais na vertical da coluna d'água na seção de medição. O ADCP foi instalado em um barco a motor que realizou diversas travessias na seção transversal do Rio Araguari na preamar (maior vazão enchente) e na baixa-mar (maior vazão vazante). As velocidades são medidas através de pulsos acústicos transmitidos a partir dos transdutores do ADCP e que, ao encontrar as partículas suspensas na água, são refletidos. A frequência do som refletido é alterada devido à velocidade das partículas. O som disperso é recebido pelos transdutores e a velocidade da partícula (e do fluido assumidas iguais) é estimada devido ao efeito Doppler.

Tabela 2: Datas das medições, marés lunares, número de travessias e duração das marés vazantes. Em setembro de 2013 e 2014, foi medida apenas a descarga líquida, por isso não foram inseridos na tabela os números de travessias.

\begin{tabular}{l|l|l|l|l}
\hline Data & Maré & $\begin{array}{l}\text { Nr de Travessias na } \\
\text { Preamar }\end{array}$ & $\begin{array}{l}\text { Nr de Travessias na } \\
\text { Baixamar }\end{array}$ & $\begin{array}{l}\text { Duração da fase da maré } \\
\text { vazante }(\mathbf{h})\end{array}$ \\
\hline 8/Junho/2013 & Sizígia & 15 & 13 & 5,3 \\
\hline 8/Junho/2014 & Quadratura & 12 & 9 & 4,7 \\
\hline 7/Dezembro/2013 & Sizígia & 28 & 11 & 4,7 \\
\hline 14/Dezembro/2014 & Quadratura & 10 & 4 & 3,5 \\
\hline 7/Setembro/2013 & Sizígia & - & - & 4,6 \\
\hline 26/Setembro/2014 & Siź́gia & - & - & 5,5 \\
\hline 8/Março/2014 & Quadratura & 15 & 5 & 5,1 \\
\hline 7/Março/2015 & Sizígia & 14 & 14 & 6,1 \\
\hline
\end{tabular}

Obtenção dos dados numéricos para a determinação dos perfis de velocidade 3D

Os dados medidos pelos ADCP e utilizados para o perfilhamento da $\mathrm{CL}$ foram extraídos através de código do MATLAB seguido por uma etapa de pós-processamento. As componentes da velocidade medidas são denominadas de Ve (velocidade longitudinal no eixo principal do escoamento ' $x$ ', na direção das abcissas, crescente para leste), Vn (velocidade no eixo das ordenadas - secundário, crescente para o norte ou sentido transversal lateral ao escoamento principal ' $y$ ') e $V z$ (velocidade na vertical, crescente para cima ' $z$ '). Para executar a análise da circulação secundária da curva estudada, executou-se uma rotação do eixo de 
orientação das velocidades, obtendo-se, assim, a velocidade no eixo do fluxo (Vx) e a velocidade perpendicular ao fluxo (Vy) - lateral. A velocidade vertical é a mesma para ambas as orientações. Vx e Vy foram calculados a partir de Ve e Vn para cada medição de acordo com as equações abaixo, onde $\theta$ é o ângulo de rotação (Equações 1 e 2):

$$
\begin{aligned}
& V_{x}=V_{e} \cdot \cos \theta-V_{n} \cdot \operatorname{sen} \theta \\
& V_{y}=V_{n} \cdot \cos \theta+V_{e} \cdot \operatorname{sen} \theta
\end{aligned}
$$

A componente $V x$ é positiva no sentido de jusante e a componente $V y$ é positiva de acordo com a regra da mão direita, ou seja, apontando o dedo indicador da mão direita para o sentido de jusante, Vy será positiva no sentido do polegar (Figura 2).

\section{Interpolação dos dados de velocidades nas seções}

Como apresentado na Tabela 2, foram feitas diversas travessias ao longo da seção estudada nas marés de preamar e baixa-mar. Para estimar registros dos perfis de cada travessia utilizou-se código Matlab para agrupar os dados por maré (preamar e baixa-mar) e por data de medição. Com o software Tecplot 360 EX 2013 R1 criou-se um prisma 3D da seção medida (Figura 3). As componentes da velocidade, após a rotação de eixo, foram adicionados a um prisma, interpolando todo o volume com o método Kriging (DAVIS, 1986). Os parâmetros de interpolação de Kriging utilizados foram amplitude de 0,30, derivada linear de valor-zero como zero. A amplitude é a distância a partir do qual os pontos a serem interpolados se tornam insignificantes e é igual à fração do comprimento da diagonal do volume 3D que contém os dados. Uma amplitude igual a zero significa que qualquer ponto não coincidente ao ponto destino é estatisticamente insignificante, enquanto uma amplitude igual a 1 implica que todos os pontos do conjunto de dados são estatisticamente significantes para cada ponto. O valor-zero é uma medida não dimensional da variância (0 a 1) da certeza do valor em um ponto. Um valor igual a zero especifica um perfeito ajuste dos pontos de origem, enquanto valores maiores permitirão alguma variância, amortecendo sua intensidade (JAMIESON et al., 2011).

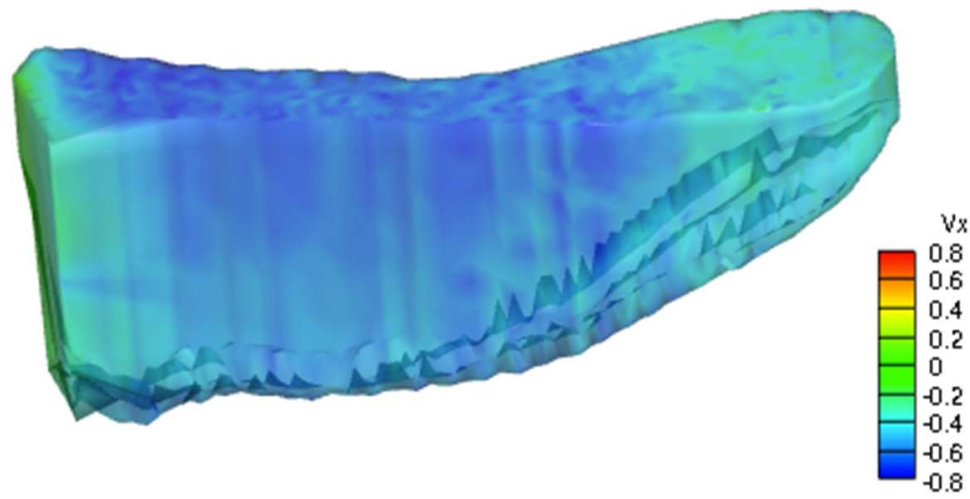

Figura 3: Prisma 3D gerado no Tecplot a partir de medições de profundidade e das componentes da velocidade com ADCP na seção estudada.

Depois de interpolados os dados das componentes das velocidades nos prismas, foram feitos cortes transversais para analisar a circulação secundária $(\mathrm{CL})$ nessa curva do Rio Araguari. Os cortes foram feitos 
buscando a coincidência da seção na maré de preamar e de baixa-mar do mesmo dia, para permitir melhor comparação da circulação secundária entre as duas fases da maré. Nos casos em que a localização espacial dos prismas gerados não permitiu coincidência no corte da seção, fez-se o corte nas seções mais próximas possíveis.

\section{Medição da vazão}

A descarga líquida $(\mathrm{Q})$ foi medida na seção de estudo com o uso de $A D C P$, durante um ciclo completo de maré semidiurna $(\approx 12,5 \mathrm{~h})$. As datas das medições são mostradas na Tabela 2 . O princípio de medição das velocidades já foi explicado anteriormente. Além da velocidade, o ADCP mede a profundidade ao longo da seção, o que permite, através da integração dos valores de velocidade na área do transecto, calcular a vazão média ( $Q$ ) e líquida $\left(Q_{\text {net }}=Q_{\text {vazante }}-Q_{\text {enchente }}\right)$ em cada travessia (MUSTE et al., 2004; DINEHART et al., 2005).

\section{RESULTADOS}

\section{Circulação lateral (CL)}

Na Figura 4 são apresentadas as informações principais sobre o padrão de circulação lateral CL na seção de estudo, bem como os detalhes da intensidade e direção das linhas de corrente de todos os períodos medidos. Cada período de medida está indicado na própria Figura 4. De acordo com a Figura 2, as linhas de corrente na maré enchente (flood tide) estão direcionadas para a margem externa da curva, enquanto na maré vazante (ebb tide), estão direcionadas para a margem interna.

June 2013 - flood tide
Qa: $-755 \mathrm{~m}^{3 / 5}$
$\mathrm{Ot} 766 \mathrm{~m}^{3 / 5}$




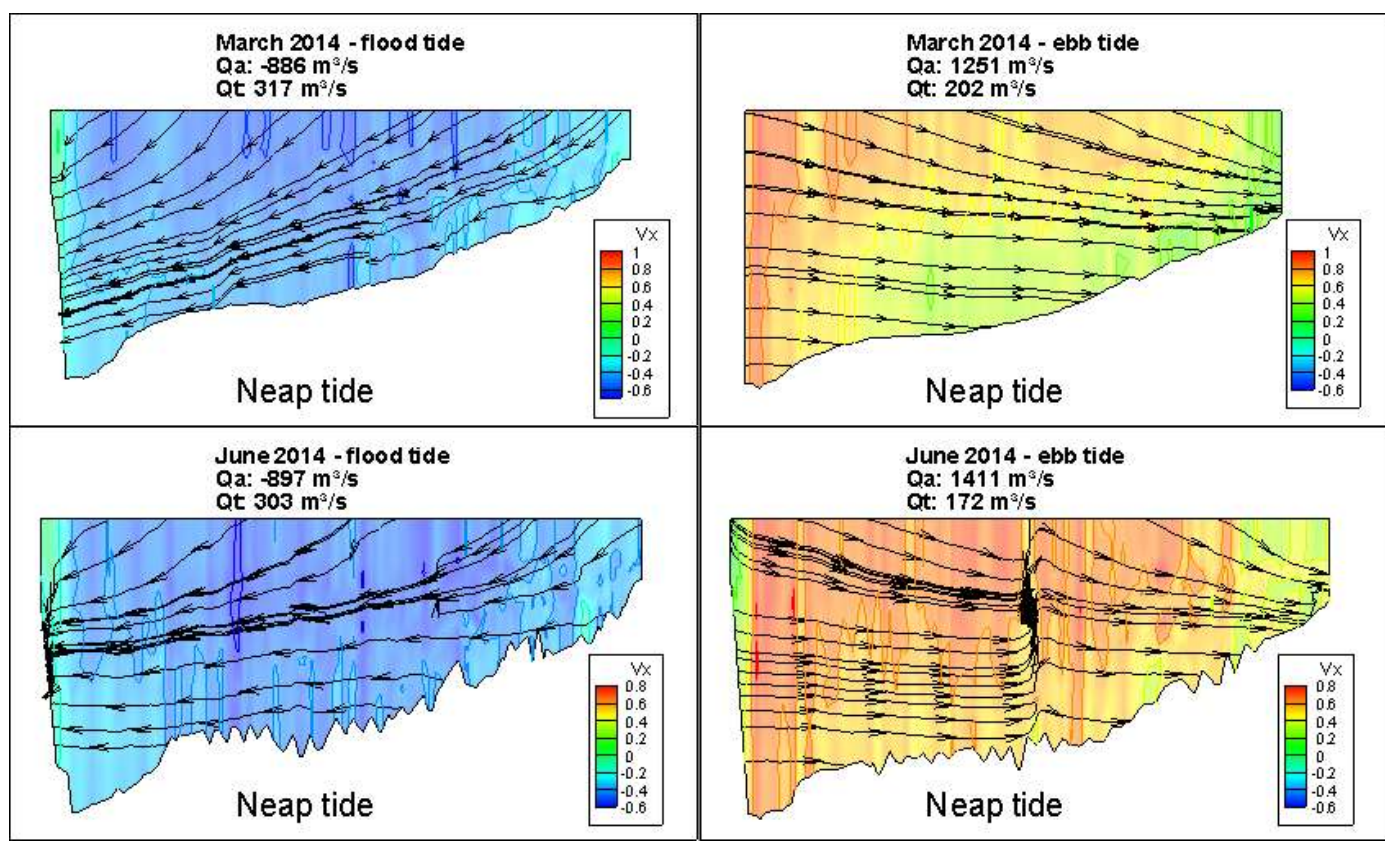

Figura 4: Comparação entre marés enchente e vazante da velocidade no sentido do fluxo (Vx) e das linhas de corrente na seção da curva do Rio Araguari de Junho/2013 a Março/15. O lado direito é a margem interna e o esquerdo, a margem externa da curva do rio.

Observando a Figura 2, a seção do Araguari fica relativamente próxima da foz do igarapé Tabaco (afluente a montante) que também é influenciado pela mesma fase da maré. Assim, o Igarapé Tabaco é um afluente relevante, contribuindo consideravelmente com a vazão total do Araguari na seção-chave (entre 0,1 a $35 \%$ da $Q_{\text {total }}$, tanto durante seu fluxo de entrada quanto no seu fluxo de saída. Para todos os períodos sazonais, o Igarapé Tabaco apresentou fluxo desaguando no Araguari durante o pico da mare enchente. De modo contrário, na maré vazante, o Igarapé Tabaco apresentava fluxo originário ou proveniente do Araguari. Contudo, isso não ocorreu nos períodos de Março e Junho/2014. Mesmo nesses 2 meses de exceção o fluxo do Igarapé Tabaco parece não ter sido capaz de alterar o padrão de circulação lateral $(\mathrm{CL})$ na seção-chave estudada. Isto é, em todos os períodos, continuou fluindo da margem externa para a margem interna e de cima para baixo. Observe os detalhes da Figura 4, onde estão destacadas as vazões Qa (Araguari) e Qt (Tabaco) e os seus respectivos sinais negativos (-) indicando sentido inverso do fluxo em relação à jusante. Em junho de 2013 surge a maior influência do Igarapé Tabaco, numa razão de Qa/Qt $\approx$ 1, não sendo mantida esta proporção nos demais períodos.

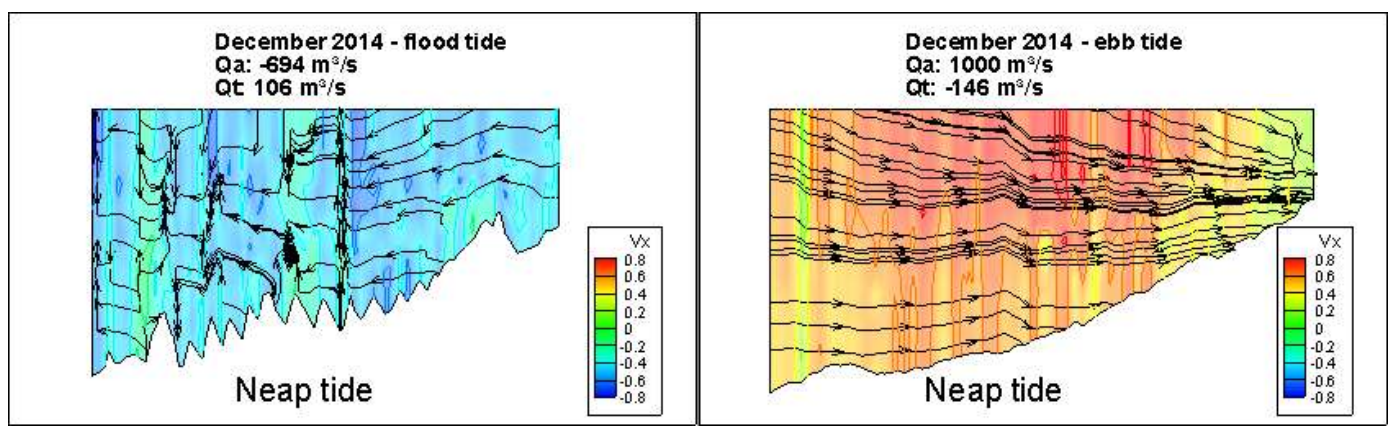






Figura 4 (continuação): Comparação entre marés enchente e vazante da velocidade no sentido do fluxo (Vx) e das linhas de corrente na seção da curva do Rio Araguari de Junho/2013 a Março/15. O lado direito é a margem interna e o esquerdo, a margem externa da curva do rio.

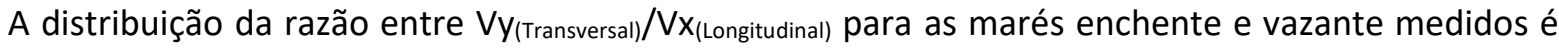
apresentada na Figura 5. A intensidade do fluxo secundário e a variabilidade são maiores na maré vazante do que na enchente para todos os períodos sazonais. A maré vazante da medição de Março/2014 resultou na menor mediana $(0,068)$ e também menor variação entre mínimo e máximo de $V y / V x$. Por outro lado, na maré enchente de Março/2015 foi medida a maior intensidade (mediana igual a 0,342) e variabilidade da intensidade do fluxo lateral. Este tipo de resposta hidrológica na seção de estudo pode ser também decorrente da ampla variabilidade climática intra-sazonal e suas as interações entre o Rio Araguari e o Rio Amazonas através de canais de marés (NIKIEMA et al., 2007).

$\mathrm{Na}$ figura 5 observamos ainda uma série significativa de valores de $\mathrm{Vy} / \mathrm{Vx}$ acima e bem superior ao limite superior da distribuição (outliers $\approx 1,5$ na escala do gráfico). Aproximadamente $2,4 \%$ dos valores medidos de $\mathrm{Vy} / \mathrm{Vx}$ são maiores que 1 . Isto é, a intensidade da velocidade lateral é mais relevante que a velocidade longitudinal, mostrando que os movimentos laterais, mesmo em curtos períodos de tempo da fase da maré ou período sazonal, tendem efetivamente a influenciar a hidrodinâmica com muita intensidade na seção específica de estudo. Esta evidência ficou mais clara durante a maré enchente de junho/2013 e março/2015.
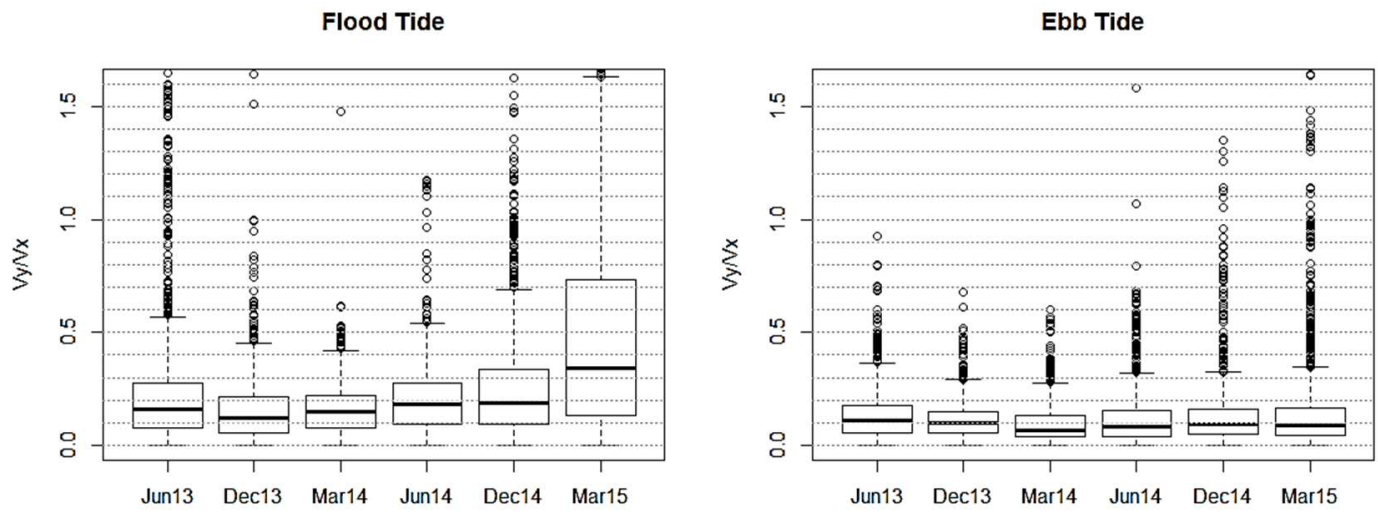

Figura 5: Distribuição da razão Vy/Vx ao longo dos períodos sazonais estudados para a maré vazante.

\section{DISCUSSÃO}

\section{Circulação lateral}

Os três principais mecanismos responsáveis pelo surgimento do fluxo secundário são: (i) a forçante 
de Ekman, que representa o equilíbrio dinâmico entre a fricção e a aceleração de Coriollis; (ii) os gradientes de pressão baroclínica na seção transversal, que surgem da advecção diferencial do gradiente de densidade longitudinal e (iii) a curvatura do fluxo, que é há muito tempo reconhecida como responsável pelo fluxo lateral helicoidal (CHANT, 2010).

Chant (2010) analisa matematicamente este fenômeno a partir de equações de conservação de momentum e de massa de cada um desses mecanismos responsáveis pelo fluxo lateral em estuário. Na seção de monitoramento estudada no Rio Araguari (Figura 2), a intensidade da aceleração de Coriollis pode ser desprezada, uma vez que a área está próxima à linha do Equador (latitude próxima a 1,3). A estratificação na coluna d'água por conta de diferenças de temperatura ou de concentração excessivas de salinidade entre diferentes profundidades foi observada em apenas dois momentos nas medições desses parâmetros, conforme apresentados nas Figuras 7 a 10. Sem essa estratificação, inexiste as condições para o aparecimento dos gradientes de densidade que permitiriam a CL na seção estudada (CHANT et al., 1997). Assim, dentre os três mecanismos apresentados, o único efetivamente observado no nosso trecho de estudo é o fator físico da curvatura do canal.

A expectativa na seção estudada era que, ao fluir no trecho curvo, o Rio Araguari apresentasse um comportamento típico esperado para esse tipo de escoamento. Isto é, com fluxo helicoidal posterior à seçãochave devido à superelevação na margem externa da curva (JAMIESON et al., 2011). A diferença de nível entre as margens externa e interna deveria criar um gradiente de pressão na seção, fazendo com que houvesse a circulação rotacional no canal através da seção (Figura 6). Este comportamento da circulação influencia a própria geomorfologia do canal com zonas de erosão e de deposição, como mostra o modelo conceitual apresentado na Figura 6. Na Figura 6, na parte mais profunda da seção, localiza-se a parte externa da curva (zona de erosão). E a parte mais rasa, localiza-se a área interna (deposição). Esse padrão dinâmico também é responsável pela abertura de tributários sempre na parte externa da curva (RILEY et al., 2012). Este elemento geomorfológico é também observado na nossa área de estudo com o Igarapé Tabaco, conectando-se ao Araguari próximo e a montante da seção-chave de monitoramento (Figura 2).

Em condições de baixa vazão, a estrutura do fluxo secundário é consistente com o padrão helicoidal tal como previsto por Chant (2002). Neste modelo, a força do fluxo lateral aumenta linearmente com a variação da maré e é consistente com a viscosidade turbulenta vertical, que, por sua vez é dependente direta da velocidade da corrente de maré (BLANCKAERT et al., 2004). Em momentos de alta descarga, a intensidade relativa do fluxo lateral $(V y / V x)$ é reduzida e sua estrutura vertical sofre uma mudança fundamental durante os ciclos de maré de sizígia e quadratura. Isto é, na maré de sizígia, é esperado que o fluxo helicoidal clássico fosse evidente, embora ficasse mais fraco do que em momentos de baixa vazão. Mas, durante marés de quadradura seria esperada para esta seção do Rio Araguari, ou seja, uma estrutura mais complexa com duas células de circulação.

Em outros estudos sobre o escoamento em trechos curvos de rios, também sob influência de maré, tem sido observado o padrão helicoidal (KALKWIJK et al., 1986; ALAEE et al., 2004; BUIJSMAN et al., 2008). Outros padrões mais complexos de circulação secundária também foram observados quando há a associação 
do efeito da curvatura e de gradientes de densidade (CHANT, 2002; BECHERER et al., 2015). Entretanto, nesse trabalho, não foi observado em nenhum dos períodos analisados, nos 2 anos de campanha hidrodinâmica, um padrão explícito de circulação helicoidal na seção do Rio Araguari.

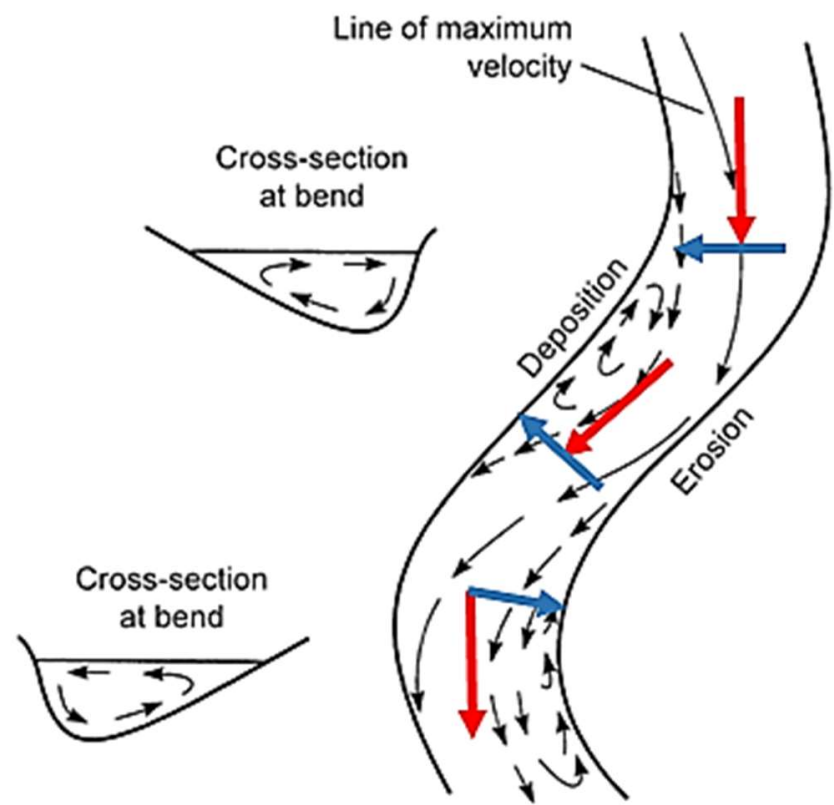

Figura 6: Fluxo helicoidal esperado em um trecho curvo de rio. Os detalhes a esquerda mostram o padrão de circulação lateral esperado para as duas curvas da figura. As setas azuis representam a velocidade secundária $\mathrm{Vy}(\mathrm{CL}) \mathrm{e}$ a vermelha, a velocidade longitudinal Vx. Fonte: Adaptado de ALLAN et al. (2007).

Os resultados obtidos a partir das componentes da velocidade medidos pelo ADCP mostraram um comportamento relativamente padrão, não importando o período sazonal (seco - dezembro, chuvoso - junho ou intermediário - março). Durante a maré vazante, quando o sentido de fluxo do Araguari escoa para o oceano, o fluxo secundário fluía em direção à margem interna (seta azul na Figura 6), mas sem a circulação pelo fundo do canal. De maneira inversa, na maré enchente, o fluxo lateral comportou-se sempre na direção da margem externa, porém sem a circulação de fundo. Em algumas das medições, foram identificadas partes da seção em que se formaram redemoinhos, como na maré enchente em dezembro/2014 (Figura 4), ou uma tensão de cisalhamento, induzindo um fluxo vertical, como observado nas marés vazante de junho/2014 (ascendente) e enchentes de dezembro/2014 (ascendente) e março/2015 (descendente) (Figura 4). Fisicamente, há fatores que inferimos estar associados com restrições do padrão helicoidal: a presença do Igarapé Tabaco a montante, cuja vazão Qt normalmente apresentava sinal oposto ao da Qa do Rio Araguari; influência da fase da maré (enchente ou vazante); e a geometria da seção-chave do rio no trecho estudado (curvatura alongada seguida de expansão abrupta).

O Igarapé Tabaco, tal qual o Araguari na seção estudada, sofre a mesma influência da maré. Portanto, apresenta reversão do fluxo ao longo do ciclo da maré praticamente na mesma fase do ciclo semidiurno (SANTOS et al., 2018). Assim, e por sua proximidade com a seção de estudo, o Igarapé Tabaco se apresenta como uma fonte de massa e energia de entrada e de saída que interfere (inibe) no fluxo tangencial ao fluxo principal pela margem esquerda do Rio Araguari (margem externa). Portanto, influenciando a hidrodinâmica do escoamento lateral na seção de monitoramento do Araguari (Figura 2). E, com base nas linhas de corrente 
da Figura 4, os fluxos de entrada e saída do Igarapé Tabaco podem atuar como inibidores evitando o desenvolvimento da estrutura tridimensional do fluxo helicoidal esperada da curva do rio (Figura 6).

Por exemplo, durante a maré enchente, o fluxo no Igarapé Tabaco estava sempre desaguando no Rio Araguari. Isso pode resultar na transferência lateral de momentum através da superfície da água, o que parece apresentar uma ação de restrição do movimento lateral que interfere na aceleração centrífuga do fluxo principal (ou lateral) em direção à margem externa. Por outro lado, na maré vazante, o fluxo de saída do Rio Araguari e entrada no Tabaco podem também servir como uma "agente de sucção que tende a intensificar a componente $V x$ em detrimento da $V y$ ", inibindo assim a superelevação no banco externo e, desta forma, evitando a formação da circulação secundária típica esperada (Figura 6). Riley et al. (2012) analisaram a estrutura do fluxo e a morfologia do canal em uma curva de rio (Rio Little Wabash) com a afluência de um tributário (Riacho Big Muddy), caso semelhante ao estudo feito no Araguari. Contudo, em região sem influência de maré. Naquele caso, entre as seções estudadas por esses autores, havia uma localizada logo a jusante da confluência do rio com o riacho em que foi observada a separação do fluxo e a formação de células de circulação helicoidal. O sentido do tributário no estudo de Riley et al. (2012) é sempre entrando no sistema fluvial, tal qual ocorre na maré enchente do Araguari. No entanto, na presente investigação não houve a formação da circulação esperada. Aqui apresentamos apenas o efeito de dois outros fatores que podem contribuir para esse fenômeno.

A maré tem um papel importante devido tanto a curvatura do rio próximo da seção quanto devido à expansão brusca da área lateral a jusante da seção (Figura 2). Ambos influenciam e direcionam a CL. Como o ciclo da maré semidiurna é de aproximadamente 12,5h, das quais entre 3,5 e a 6,1 eram escoando em direção ao oceano (Tabela 2) e, no tempo restante, o escoamento fluía no sentido contrário e em regime transiente. Este tipo de sistema típico de rios de estuários da Amazônia tende a ser também responsável pela inibição da formação da circulação lateral do fluxo na seção. Como a vazão varia continuamente ao longo do ciclo de maré, os vetores da velocidade tendem a seguir o mesmo comportamento, ou seja, são modificados a cada instante deste ciclo. E esse efeito, que deveria ser avaliado à luz de em conjunto de outros fatores aqui apresentados, tem impedido a formação do fluxo helicoidal.

O terceiro fator que apontamos como inibidor da circulação secundária na seção de estudo é a própria geometria do Rio Araguari no trecho estudado. Como é apresentado na Figura 2, o transecto estudado está bem próximo do trecho em que ocorre uma expansão da largura do canal. A largura da seção estudada é de aproximadamente $850 \mathrm{~m}$. A partir dela, o canal se expande e a largura do rio atinge $1.750 \mathrm{~m}$ cerca de $2.900 \mathrm{~m}$ a jusante. Esse aumento da largura no trecho é da ordem de $310 \mathrm{~m} / \mathrm{km}$ ocorrendo a partir na margem direita (parte interna da curva). No escoamento em direção ao oceano (maré vazante), o fluxo sofre uma expansão ao passar pela seção de estudo aumentando a intensidade da turbulência lateral (espalhamento do escoamento) e levando à separação do fluxo, que fica evidente pela formação do banco de sedimentos que se forma logo após a seção (Figura 2).

Esse resultado está de acordo com o previsto por Wang et al. (2016) a partir de dados experimentais em laboratório. No sentido inverso, em que o rio corre para montante, na maré, enchente, ocorre o inverso, 
ou uma redução brusca da área de escoamento por causa da constrição do canal, provocando um efeito hidrodinâmico contrário ao caso anterior de vazante (aceleração do fluxo longitudinal). Consequentemente, também ocorre um aumento da velocidade no trecho mais estreito do rio (aceleração), aumentando a turbulência e a contração do escoamento e até a intensificação da componente Vy devido à influência lateral proveniente do trecho mais largo do canal.

Como pode ser observado na Figura 5, tal efeito resulta em maior intensidade da velocidade através do canal $(V y)$ em relação à velocidade ao longo do canal $(V x)$. Portanto, a razão $V y / V x$ maior na maré enchente do que na vazante ocorreu para todos os períodos sazonais estudados. Tipicamente, a razão entre a Vy e Vx é menor que $10 \%$ na maior parte do tempo. Mas esse valor pode ser maior que 1,5 (150\%) por conta dos gradientes laterais de velocidade, salinidade, turbidez e outros traçadores (CHANT, 2010).

Na seção estudada, como mostrado na Figura 5, Vy/Vx ficou abaixo dos $10 \%$ em quase todas as medições realizadas na maré vazante. A única exceção foi em Junho/2013, cujo valor foi de 11,2\%. Entretanto, para a maré enchente, em nenhum dos períodos a relação ficou abaixo dos $10 \%$, sendo que em Março/2015, a mediana foi superior a $34 \%$ e com significativa variabilidade $(p<0,05)$, com valor do 3 o interquartil superior a 160\%, mostrando como a turbulência lateral foi maior e mais intensa, em acordo com o dado de vazão que também foi o maior na enchente nesse período em relação aos demais (Figuras 4 e 5).

\section{CONCLUSÕES}

O presente estudo apresenta as seguintes conclusões relacionadas com os resultados hidrodinâmicos: 1) Aceita-se a hipótese de não formação do perfil hidrodinâmico helicoidal padrão esperado para a CL. Contudo, na fase de maré vazante (jusante), em média, as linhas de corrente $(\Omega)$ ocorreram da margem externa para a interna e da superfície para o fundo, sem um padrão evidente da CL. E na fase de maré enchente, ocorreu um padrão de CL oposto, com o fluxo secundário fluindo da margem interna para a externa e descendente para o fundo do rio; 2) Aceita-se a hipótese de que as intensidades dos fluxos secundários (CLs) estão correlacionadas com a fase da maré, onde o menor valor de Vy/Vx ocorreu na maré vazante $(\approx 0,091)$ e o maior na enchente $(\approx 0,169)$, sugerindo uma maior intensidade do fluxo secundário durante a fase de maré enchente. Assim, foram considerados relevantes para o comportamento atípico do escoamento (1) a estratificação de parâmetros da qualidade da água (2) a presença de um afluente próximo da seção-chave e (3) a expansão da largura do rio, (4) a inversão de fluxo devido à da maré; 3) Aceita-se a hipótese de potencial existência de correlação entre $C L(o u V y / V x)$ e parâmetros da qualidade da água, variando com a fase da maré. Contudo, tal correlação não é genérica, ocorrendo somente próximo da superfície ou do fundo do canal. Isso demonstra que a CL é um parâmetro relevante para estudos de monitoramento da qualidade da água e gestão e conservação nesses ecossistemas aquáticos estuarinos; 4) Estas informações demonstram que os movimentos de $\mathrm{CL}$ podem não somente definem as características geomorfológicas dos canais, mas também influenciam na dinâmica da variação da paisagem em geral da bacia hidrográfica, devendo ser levados em consideração nos estudos eco-hidrológicos na região estuarina amazônica, principalmente em processos de modelagem hidrodinâmica e qualidade da água. 


\section{REFERÊNCIAS}

ALAEE, M. J.; IVEY, G.; PATTIARATCHI, C.. Secondary circulation induced by flow curvature and Coriolis effects around headlands and islands. Ocean Dynamics, v.54, n.1, p.27-38, 2004. DOI: http://doi.org/10.1007/s10236-003$\underline{0058-3}$

ABREU, C. H. M.; BARROS, M. L. C.; BRITO, D. C.; TEIXEIRA, M. R.; CUNHA, A. C.. Hydrodynamic modeling and simulation of water residence time in the Estuary of the Lower Amazon River. Water, v.12, p.1/60-30, 2020. DOI: http://dx.doi.org/10.3390/w12030660

ALLAN, D.; CASTILLO, M.. Stream Ecology: Structure and Function of Running Waters. 2 ed. Dordrecht: Springer, 2007.

BECHERER, J.; STACEY, M. T.; UMLAUF, L.; BURCHARD, H.. Lateral Circulation Generates Flood Tide Stratification and Estuarine Exchange Flow in a Curved Tidal Inlet. Journal of Physical Oceanography, v.45, n.3, p.638-656, 2015. DOI: http://doi.org/10.1175/JPO-D-14-0001.1

BLANCKAERT, K.. Hydrodynamic processes in sharp meander bends and their morphological implications. J. Geophys. Res. Earth Surf., v.116, p.1-22, 2011. DOI:

http://doi.org/10.1029/2010JF001806

BLANCKAERT, K.; VRIEND, H. J.. Secondary flow in sharp open-channel bends. J. Fluid Mech., v.498, p.353-380, 2004. DOI: http://doi.org/10.1017/S0022112003006979

BLETTLER, M.; AMSLER, M.; DRAGO, I. E.; MARCHESE, M.. Effects of stream hydraulics and other environmental variables on density of Narapa bonettoi (Oligochaeta) in the Paraná River system. River Res. Appl., v.24, p.1124-1140, 2008. DOI: http://doi.org/10.1002/rra.1115

BUIJSMAN, M. C.; RIDDERINKHOF, H.. Variability of secondary currents in a weakly stratified tidal inlet with low curvature. Continental Shelf Research, v.28, n.14, p.17111723, 2008. DOI: http://doi.org/10.1016/j.csr.2008.04.001

CARTHEY, A. J. R.; FRYIRS, K. A.; RALPH, T. J.; BU, H.; LEISHMAN, M. R.. How seed traits predict floating times: A biophysical process model for hydrochorous seed transport behaviour in fluvial systems. Freshwater Biology, v.61, p.1931, 2016. DOI: http://doi.org/10.1111/fwb.12672

CHANT, R. J.; WILSON, R. E.. Secondary circulation in a highly stratified estuary. Journal of Geophysical Research, v.102, n.C10, p.23207, 1997. DOI: http://doi.org/10.1029/97JC00685

CHANT, R. J.. Secondary circulation in a region of flow curvature: Relationship with tidal forcing and river discharge. J. Geophys. Res., v.107, p.1-11, 2002. DOI: http://doi.org/10.1029/2001JC001082

CHANT, R.. Secondary Flows. In: VALLE-LEVINSON, A.. Contemporary Issues in Estuarine Physics. New York: Cambridge University Press, 2010. p.100-124.

CUNHA, A. C.; MUSTIN, K.; SANTOS, E. S.; SANTOS, É. W. G.; GUEDES, M. C.; CUNHA, H. F. A.; STERNBERG, L. S. L.. Hydrodynamics and seed dispersal in the lower Amazon. Freshwater Biology, p.1-9, 2017. DOI: http://doi.org/10.1111/fwb.12982
CUNHA, A. C.; STERNBERG, L. S. L.. Using stable isotopes 180 and $2 \mathrm{H}$ of lake water and biogeochemical analysis to identify factors affecting water quality in four estuarine Amazonian shallow lakes. Hydrological Processes, v.32, p.1188-1201, 2018. DOI: http://dx.doi.org/10.1002/hyp.11462

DAVIS, J. C.. Statistics and data analysis in geology. 2 ed. New York: John Wiley \& Sons Ltd., 1986.

DINEHART, R. L.; BURAU, J. R.. Repeated surveys by acoustic Doppler current profiler for flow and sediment dynamics in a tidal river. Journal of Hydrology, v.314, n.1, p.1-21, 2005. DOI: http://dx.doi.org/10.1016/j.jhydrol.2005.03.019

DOLÉDEC, S.; LAMOUROUX, N.; FUCHS, U.; MÉRIGOUX, S. Modelling the hydraulic preferences of benthic macroinvertebrates in small European streams. Freshwater Biology, v.52, p.145-164, 2007. DOI: http://dx.doi.org/10.1111/i.1365-2427.2006.01663.x

FAGHERAZZI, S.; GABET, E. J.; FURBISH, D. J.. The effect of bidirectional flow on tidal channel planforms. Earth Surf. Process. Landforms, v.29, p.295-309, 2004. DOI: http://dx.doi.org/10.1002/esp.1016

FRIEDRICHS, C. T.; HAMRICK, J. M.. Effects of channel geometry on cross sectional variations in along channel velocity in partially stratified estuaries. Buoyancy Effects on Coastal and Estuarine Dynamics, v.53, p.283-300, 1996. DOI: http://doi.org/10.1029/CE053p0283

HOWE, H. F.; SMALLWOOD, J.. Ecology of seed dispersal. Annual Review of Ecology and Systematics, v.13, p.201-228, 1982.

JAMIESON, E. C.; RENNIE, C. D.; JACOBSON, R. B.; TOWNSEND, R. D.. 3-D flow and scour near a submerged wing dike: ADCP measurements on the Missouri River. Water Resource Research, v.47, p.1-20, 2010. DOI: http://doi.org/10.1029/2010WR010043

KALKWIJK, J. P. T.; BOOIJ, R.. Adaptation of secondary flow in nearly-horizontal flow. Journal of Hydraulic Research, v.24, n.1, p.19-37, 1986

KASHYAP, S.; CONSTANTINESCU, G.. Influence of Channel Aspect Ratio and Curvature on Flow, Secondary Circulation, and Bed Shear Stress in a Rectangular Channel Bend. Journal of Hydraulics, v.138, p.1045-1060, 2012. DOI: http://doi.org/10.1061/(ASCE)HY.1943-7900.0000643

KAWANISI, K.. Structure of turbulent flow in a shallow tidal estuary. Journal of Hydraulic Engineering, v.130, p.360-370 2004. DOI: http://doi.org/10.1061/(ASCE)07339429(2004)130:4(360)

KESHAVARZI, A.; HAMIDIFAR, H.; BALL, J.. Bed morphology in vegetated estuarine river with mild-curved meander bend. Hydrological Sciences Journal, v.61, n.11, p.2033-2049, 2015. DOI: http://doi.org/10.1080/02626667.2015.1081201

KIMMERER, W. J.. Effects of freshwater flow on abundance of estuarine organisms: physical effects or trophic linkages? Marine Ecology Progress Series, v.243, p.39-55, 2002. DOI: http://doi.org/10.3354/meps243039 
KUPILAS, B.; HERING, D.; LORENZ, A. W.; KNUTH, C.; GÜCKER, B.. Hydromorphological restoration stimulates river ecosystem metabolism. Biogeosciences, v.14, p.1989-2002, 2017. DOI: http://doi.org/10.5194/bg-14-1989-2017

MARION-ZARAMELLA, M. A.. Effects of velocity gradients and secondary flow on the dispersion of solutes in a meandering channel. Journal of Hydraulic Engineering, v.132, p.1296-1302, 2006. DOI:

http://doi.org/10.1061/(ASCE)07339429(2006)132:12(1295)

MUSTE, M.; YU, K.; SPASOJEVIC, M.. Practical aspects of $A D C P$ data use for quantification of mean river flow characteristics; Part I: moving-vessel measurements. Flow Measurement and Instrumentation, v.15, n.1, p.1-16, 2004. DOI: http://doi.org/10.1016/i.flowmeasinst.2003.09.001

NIKIEMAA, O.; DEVENONA, J.-L.; BAKLOUTIB, M.. Review Numerical modeling of the Amazon River plume.

Continental Shelf Research, v.27, p.873-899, 2007. DOI: http://doi.org/10.1016/j.csr.2006.12.004

NILSSON, C.; BROWN, R. L.; JANSSON, R.; MERRITT, D. M.. The role of hydrochory in structuring riparian and wetland vegetation. Biological Reviews, v.85, p.837-858, 2010. DOI: http://doi.org/10.1111/j.1469-185X.2010.00129.x

OLIVEIRA, L. L.; CUNHA, A. C.; JESUS, E. S.; BARRETO, N. J. C.. Características Hidroclimáticas da Bacia do Rio Araguari. In: CUNHA, A. C.; SOUZA, E. B.; CUNHA, H. F. A.. Tempo, clima e recursos hídricos: resultados do projeto REMETAP no Estado do Amapá. IEPA, 2010. p.216.

QIN, C.; SHAO, X.; ZHOU, G.. Comparison of Two Different Secondary Flow Correction Models for Depth-averaged Flow Simulation of Meandering Channels. Procedia Eng., v.154, p.412-419, 2016. DOI:

http://doi.org/10.1016/i.proeng.2016.07.507

RAZAZ, M.; KAWANISI, K.; NISTOR, I.. Tide-driven controls on maximum near-bed floc size in a tidal estuary. J. HydroEnvironment Res., v.9, p.465-471, 2015. DOI: http://doi.org/10.1016/j.jher.2014.04.001

RILEY, J. D.; RHOADS, B. L.. Flow structure and channel morphology at a natural confluent meander bend. Geomorphology, v.163-164, p.84-98, 2012. DOI: http://doi.org/10.1016/i.geomorph.2011.06.011

SANTOS, E. S.; CUNHA, A. C.; CUNHA, E. D. S.. Análise espaço-sazonal da qualidade da água na zona flúvio-marinha do Rio Araguari-Amazônia Oriental-Brasil. Revista Brasileira de Recursos Hídricos, v.19, n.3, p.215-226, 2014. DOI: http://doi.org/10.21168/rbrh.v19n3.p215-226

SANTOS, E. S.; LOPES, P. P. P.; NASCIMENTO, O. O.; PEREIRA, H. H. S.; COLLIN, R.; STERNBERG, L. S. L.; CUNHA, A. C.. The impact of channel capture on estuarine hydromorphodynamics and water quality in the Amazon delta. Science of the Total Environment, v.624, p.887-899, 2018. DOI: http://doi.org/10.1016/j.scitotenv.2017.12.211
SANTOS, P. V. C. J.. Influência hidroclimática sobre a biodiversidade de peixes e insetos aquáticos no Baixo Curso de uma Bacia da Região Amazônica. Universidade Federal do Maranhão, 2017.

SHIELDS, F. D.; RIGBY, J. R.. River habitat quality from river velocities measured using acoustic Doppler current profiler. Environmental Management, v.36, n.4, p.565-75, 2005. DOI: http://doi.org/10.1007/s00267-004-0292-6

SUKHODOLOV, A. N.; BLETTLER, M.; ZHANG, J.; SUKHODOLOVA, T.; NÜTZMANN, G.. A study of flow dynamics and implications for benthic fauna in a meander bend of a lowland river. Journal of Hydraulic Research, v.53, p.488-504, 2015. DOI: http://doi.org/10.1080/00221686.2015.1055598

VOROSMARTY, C. J.; MCINTYRE, P. B.; GESSNER, M. O.; DUDGEON, D.; PRUSEVICH, A.; GREEN, P.; DAVIES, P. M. Global threats to human water security and river biodiversity. Nature, v.467, n.7315, p.555-561, 2010. DOI: http://dx.doi.org/10.1038/nature09440

WANG, X.; WANG, B.; LIU, X.; ZHANG, L.. Effects of river width changes on flow characteristics based on flume experiment. Journal of Mountain Science, v.13, n.2, p.361368, 2016. DOI: http://doi.org/10.1007/s11629-014-3265-0

WARD, N. D.; KEIL, R. G.; MEDEIROS, P. M.; BRITO, D. C.; CUNHA, A. C.; DITTMAR, T.; YAGER, P. L.; KRUSCHE, A. V.; RICHEY, J. E.. Degradation of terrestrially derived macromolecules in the Amazon River. Nature Geoscience (Print), v.6, p.530-533, 2013. DOI: http://doi.org/10.1038/ngeo1817

WARD, N. D.; KRUSCHE, A. V.; SAWAKUCHI, H. O.; BRITO, D. C.; CUNHA, A. C.; MOURA, J. M. S.; SILVA, R.; YAGER, P. L.; KEIL, R. G.; RICHEY, J. E.. The compositional evolution of dissolved and particulate organic matter along the lower Amazon River-Óbidos to the ocean. Marine Chemistry (Print), v.177, p.244-256, 2015. DOI: http://doi.org/10.1016/j.marchem.2015.06.013

WARD, N. D.; BIANCHI, T. S.; SAWAKUCHI, H. O.; GAGNEMAYNARD, W.; CUNHA, A. C.; BRITO, D. C.; NEU, V.; DE MATOS VALERIO, A.; DA SILVA, R.; KRUSCHE, A. V.; RICHEY, J. E.; KEIL, R. G.. The reactivity of plant-derived organic matter and the potential importance of priming effects along the lower Amazon River. Journal of Geophysical Research: Biogeosciences, v.121, p.1522-1539, 2016. DOI: http://doi.org/10.1002/2016JG003342

WEBSTER, I. T.. The hydrodynamics and salinity regime of a coastal lagoon - The Coorong, Australia - Seasonal to multidecadal timescales. Estuarine, Coastal and Shelf Science, v.90, n.4, p.264-274, 2010. DOI: http://doi.org/10.1016/j.ecss.2010.09.007

ZĂINESCU, F.; VESPREMEANU-STROE, A.; ANTHONY, E.; TĂTUI, F.; PREOTEASA, F.; MATEESCU, R.. Flood deposition and storm removal of sediments in front of a deltaic waveinfluenced river mouth. Marine Geology, v.417, p.106015, 2019, DOI: http://doi.org/10.1016/j.margeo.2019.106015

A CBPC - Companhia Brasileira de Produção Científica (CNPJ: 11.221.422/0001-03) detém os direitos materiais desta publicação. Os direitos referem-se à publicação do trabalho em qualquer parte do mundo, incluindo os direitos às renovações, expansões e disseminações da contribuiç̃o, bem como outros direitos subsidiários. Todos os trabalhos publicados eletronicamente poderão posteriormente ser publicados em coletâneas impressas sob coordenação da Sustenere Publishing, da Companhia Brasileira de Produção Científica e seus parceiros autorizados. Os (as) autores (as)

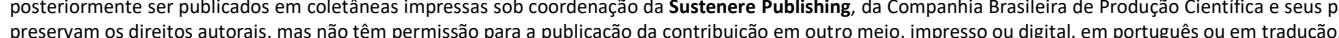

